\title{
On the Ramanujan AGM Fraction, I: The Real-Parameter Case
}

\author{
J. Borwein, R. Crandall, and G. Fee
}

\section{CONTENTS}

1. Introduction

2. Preliminaries

3. Sech-Elliptic Forms

4. Relations for $\mathcal{R}(a)$

5. The $\mathcal{R}$ Function at Rational Agruments

6. Transformation of $\mathcal{R}_{1}(a, b)$

7. Convergence Results for Real Parameters

8. A Uniformly Convergent Algorithm

9. Complex Parameters

Acknowledgments

References
2000 AMS Subject Classification: Primary 44-A20;

Secondary 33C05, 11J70

Keywords: Continued fractions, theta functions, elliptic integrals, hypergeometric functions, special functions
The Ramanujan AGM continued fraction is a construct

$$
\mathcal{R}_{\eta}(a, b)=\frac{a}{\eta+\frac{b^{2}}{\eta+\frac{4 a^{2}}{\eta+\frac{9 b^{2}}{\eta+} \cdot}}}
$$

enjoying attractive algebraic properties, such as a striking arithmetic-geometric mean (AGM) relation and elegant connections with elliptic-function theory. But the fraction also presents an intriguing computational challenge. Herein we show how to rapidly evaluate $\mathcal{R}$ for any triple of positive reals $a, b, \eta$. Even in the problematic scenario when $a \approx b$ certain transformations allow rapid evaluation. In this process we find, for example, that when $a \eta=b \eta=$ a rational number, $\mathcal{R}_{\eta}$ is essentially an $L$ series that can be cast as a finite sum of fundamental numbers. We ultimately exhibit an algorithm that yields $D$ good digits of $\mathcal{R}$ in $O(D)$ iterations where the implied big- $O$ constant is independent of the positive-real triple $a, b, \eta$. Finally, we address the evidently profound theoretical and computational dilemmas attendant on complex parameters, indicating how one might extend the AGM relation for complex parameter domains.

\section{INTRODUCTION}

In Entry 12 of Chapter 18 of Ramanujan's Second Notebook [Berndt 99b] one finds the beautiful construct

$$
\mathcal{R}_{\eta}(a, b)=\frac{a}{\eta+\frac{b^{2}}{\eta+\frac{4 a^{2}}{\eta+\frac{9 b^{2}}{\eta+} \cdot}}}
$$

which we interpret - in most but not all of the present treatment-for real $a, b, \eta>0$. Remarkably, for the indicated parameter space, $\mathcal{R}$ satisfies an AGM relation

$$
\mathcal{R}_{\eta}\left(\frac{a+b}{2}, \sqrt{a b}\right)=\frac{\mathcal{R}_{\eta}(a, b)+\mathcal{R}_{\eta}(b, a)}{2} .
$$


This relation is one of many expedients we shall develop when computing $\mathcal{R}_{\eta}$. It will turn out that the computationally difficult cases can be summarized in the phrase " $b$ is near to $a$," including the case $a=b$. What we shall eventually exhibit is a computational algorithm that is uniformly of geometric convergence (i.e., the error after $n$ steps is $O\left(\theta^{-n}\right)$ for some real $\left.\theta>1\right)$ across the entire positive quadrant $a, b>0$. Along the way, we find attractive identities, such as the expression of any $\mathcal{R}_{1}(r, r)$, where $r$ is rational, as a finite series of fundamental numbers. Finally, we consider complex $a, b$ and note the considerable difficulties in such analysis; accordingly, we prove results and posit various conjectures pertaining to fraction convergence and the domain of validity for the AGM relation (1-2).

We would be remiss in not adding this perspective: the present research began when the authors realizedvia numerical experimentation - that $\mathcal{R}_{1}(1,1)$ "seemed to be" the number $\log 2$. Such is the value of experiment: one can be led thereby into deep waters.

\section{PRELIMINARIES}

An initial observation is that

$$
\mathcal{R}_{\eta}(a, b)=\mathcal{R}_{1}(a / \eta, b / \eta),
$$

as can be formally inferred by cancellation of the $\eta$ elements down through the continued-fraction form. Such manipulations are valid when the continued fraction $\mathcal{R}_{\eta}$ converges. One way to prove convergence - at least for positive, real $a$ and $b$-is to put the entity $a / \mathcal{R}_{1}$ in RCF (reduced continued fraction) form, meaning

$$
\begin{aligned}
& \mathcal{R}_{1}(a, b)=\frac{a}{\left[A_{0} ; A_{1}, A_{2}, A_{3}, \ldots\right]} \\
& A_{0}+\frac{a}{A_{1}+\frac{1}{A_{2}+\frac{1}{A_{3}+} \cdot}}
\end{aligned}
$$

where the elements $A_{i}$ are all positive reals. (We take the more restricted, classical mnemonic SCF (simple continued fraction) to denote the instance of an $\mathrm{RCF}$ where each $A_{i}$ is a positive integer; in our present case, though, the $A_{i}$ are not generally integers.) Inspection of Ramanujan's pattern for $\mathcal{R}$ reveals that the RCF elements can be given explicitly:

$$
A_{n}=\frac{n !^{2}}{(n / 2) !^{4}} 4^{-n} \frac{b^{n}}{a^{n}} \sim \frac{2}{\pi n} \frac{b^{n}}{a^{n}}, n \text { even, }
$$

$$
A_{n}=\frac{((n-1) / 2 !)^{4}}{n !^{2}} 4^{n-1} \frac{a^{n-1}}{b^{n+1}} \sim \frac{\pi}{2 a b n} \frac{a^{n}}{b^{n}}, \quad n \text { odd. }
$$

Here we have indicated also the asymptotic behavior of the $A_{n}$. This element representation leads immediately to the following:

Theorem 2.1. For any positive real pair $a, b$, the continued fraction $\mathcal{R}_{1}(a, b)$ converges (to a finite limit).

Remark 2.2. In continued-fraction theory there is the concept of converging on the extended complexes, including $\infty$ (see Section 9), so we have emphasized a finite limit in Theorem 2.1.

Proof: It is known that an RCF with positive real elements converges to a finite limit iff $\sum A_{i}$ diverges (this is the Seidel-Stern theorem [Khintchine 64, Lorentzen and Waadeland 92]). In our case, such divergence is evident for any choice of real $a, b>0$.

Indeed, the divergence of $\sum A_{i}$ is only logarithmic for $a=$ $b$, and this is a true indication of slow convergence (we wax more quantitatively later). Sure enough, our interest in the computational aspect started with the question of how to rapidly evaluate

$$
\mathcal{R}(a):=\mathcal{R}_{1}(a, a)
$$

for positive real $a$ and, thereby, to prove some suspected identities. We shall encounter later a different continued fraction for $\mathcal{R}(a)$, as well as other computationally efficient constructs.

\section{SECH-ELLIPTIC FORMS}

Using connections between standard Jacobi theta functions $\theta_{2}, \theta_{3}$ and elliptic integrals, we can establish various results; the wonderful sech identities to follow stem from classical work of Rogers, Stieltjes, Preece, and, of course, Ramanujan [Berndt 99b] in which one may find the earlier work detailed. We start with the following theorem.

Theorem 3.1. For real $y, \eta>0$ and $q:=e^{-\pi y}$ we have

$$
\begin{aligned}
& \eta \sum_{k \in D} \frac{\operatorname{sech}(k \pi y / 2)}{\eta^{2}+k^{2}}=\mathcal{R}_{\eta}\left(\theta_{2}^{2}(q), \theta_{3}^{2}(q)\right), \\
& \eta \sum_{k \in E} \frac{\operatorname{sech}(k \pi y / 2)}{\eta^{2}+k^{2}}=\mathcal{R}_{\eta}\left(\theta_{3}^{2}(q), \theta_{2}^{2}(q)\right),
\end{aligned}
$$

where $D, E$ denote respectively the odd, even integers. Accordingly, the Ramanujan AGM identity (1-2) holds for positive triples $\eta, a, b$. 
Remark 3.2. The following proof for the AGM conclusion has been interpreted for certain complex $a$ and $b$ sometimes incorrectly in the literature (see Section 9). For the moment, we are stating the AGM part of Theorem 3.1 for positive reals $a$ and $b$, with attention paid to complex parameters later in this work. In the treatment of Berndt (see proof) the classical work of Stieltjes and Rogers is seen to imply that the relevant (real-parameter) continued fractions do converge and equal the sech series via certain integrals of Jacobi-elliptic functions. Similarly, [Wall 48] has fraction-integral equality theorems. Such results have not been completely applied for complex parameters, and this gives rise to a difficult, conjectural scenario we address in a later section.

Proof: The sech relations are proved - with somewhat different but equivalent notation - in Berndt's treatment [Berndt 99b, Vol II, Ch. 18] of Ramanujan's Notebooks. As for the AGM identity, observe that for $0<b<a$ the assignments

$$
\begin{gathered}
\theta_{2}(q)^{2} / \theta_{3}(q)^{2}:=b / a \\
\eta:=\theta_{2}(q)^{2} / b
\end{gathered}
$$

are possible (since $b / a \in[0,1)$, see [Borwein and Borwein 87]), implicitly define $q, \eta$, and, together with the Jacobi identities

$$
\begin{gathered}
\theta_{2}(q)^{2}+\theta_{3}(q)^{2}=\theta_{3}(\sqrt{q})^{2}, \\
2 \theta_{2}(q) \theta_{3}(q)=\theta_{2}(\sqrt{q})^{2},
\end{gathered}
$$

and the sech sums in the theorem, yield

$$
\begin{array}{r}
\mathcal{R}_{1}\left(\theta_{3}(q)^{2} / \eta, \theta_{2}(q)^{2} / \eta\right)+\mathcal{R}_{1}\left(\theta_{2}(q)^{2} / \eta, \theta_{3}(q)^{2} / \eta\right) \\
=2 \mathcal{R}_{1}\left(\theta_{3}(\sqrt{q})^{2} /(2 \eta), \theta_{2}(\sqrt{q})^{2} /(2 \eta)\right) .
\end{array}
$$

Thus, the AGM identity (1-2) holds for any positive reals $a>b$. The case $0<a<b$ is handled symmetrically, starting with $\theta_{2}(q)^{2} / \theta_{3}(q)^{2}:=a / b$, or via the $\theta$ transform.

These sech series can be used in turn to establish two evaluation series involving the standard elliptic integral $K$ :

Theorem 3.3. In what follows we intend $\mathrm{K}:=\mathrm{K}(k)$ and $\mathrm{K}^{\prime}:=\mathrm{K}\left(k^{\prime}\right)$ with $k^{\prime}:=\sqrt{1-k^{2}}$. For real $0<b<a$ and $k:=b / a$, we have

$$
\mathcal{R}_{1}(a, b)=\frac{\pi a \mathrm{~K}}{2} \sum_{n \in Z} \frac{\operatorname{sech}\left(n \pi \frac{\mathrm{K}^{\prime}}{\mathrm{K}}\right)}{\mathrm{K}^{2}+\pi^{2} a^{2} n^{2}} .
$$

On the other hand, for $0<a<b$ and $k:=a / b$, we have

$$
\mathcal{R}_{1}(a, b)=2 \pi b \mathrm{~K} \sum_{n \in D} \frac{\operatorname{sech}\left(n \pi \frac{\mathrm{K}^{\prime}}{2 \mathrm{~K}}\right)}{4 \mathrm{~K}^{2}+\pi^{2} b^{2} n^{2}} .
$$

Proof: The two series follow from the assignments $\theta_{3}^{2}(q) / \eta:=\max (a, b)$ and $\theta_{2}^{2}(q) / \eta:=\min (a, b)$ and the classical relations

$$
e^{-\pi K^{\prime} / K}=q, \quad K=\frac{\pi}{2} \theta_{3}(q)^{2}
$$

inserted into the appropriate sech identities from Theorem 3.1.

The sech-elliptic series (3-1) and (3-2) do allow for rapid computation of $\mathcal{R}_{1}(a, b)$ when $b$ is not close to $a$. Indeed, to get $D$ good digits for $\mathcal{R}_{1}$, one requires $O\left(D \mathrm{~K} / \mathrm{K}^{\prime}\right)$ summands. So, yet another motive for the present analysis was the problem of slow convergence of the sech-elliptic form for $b \approx a$.

Note that we also have attractive evaluations such as

$$
\mathcal{R}_{1}\left(1, \frac{1}{\sqrt{2}}\right)=\frac{\pi}{2} \mathrm{~K}(1 / \sqrt{2}) \sum_{n \in \mathcal{Z}} \frac{\operatorname{sech}(n \pi)}{\mathrm{K}^{2}(1 / \sqrt{2})+n^{2} \pi^{2}},
$$

where we remind ourselves that $\mathrm{K}(1 / \sqrt{2})$ = $\Gamma^{2}(1 / 4) /(4 \sqrt{\pi}) \quad$ [Borwein and Borwein 87], with similar series for $\mathcal{R}_{1}\left(1, k_{N}\right)$ at the $N$ th singular value, as discussed in [Borwein et al. 04]. A similar relation for $\mathcal{R}_{1}\left(\frac{1}{\sqrt{2}}, 1\right)$ obtained via (3-2), and via the AGM relation (1-2), yields in turn the oddity

$\mathcal{R}_{1}\left(\frac{1+\sqrt{2}}{2 \sqrt{2}}, \frac{1}{2^{1 / 4}}\right)=\pi \mathrm{K}(1 / \sqrt{2}) \sum_{n \in \mathcal{Z}} \frac{\operatorname{sech}(n \pi / 2)}{4 \mathrm{~K}^{2}(1 / \sqrt{2})+n^{2} \pi^{2}}$.

\section{RELATIONS FOR $\mathcal{R}(a)$}

Recalling that $\mathcal{R}(a):=\mathcal{R}_{1}(a, a)$, we next derive relations for the problematic cases $b=a$. Interpreting $(3-1)$ as a Riemann-integral relation in the limit $b \rightarrow a^{-}$, we have (for $a>0$ ) a slew of relations involving the digamma function $\psi:=\Gamma^{\prime} / \Gamma$ [Stromberg 81, Abramovitz and Stegun 70$]$ and the Gauss-hypergeometric $F$, here presented in an order that can be serially derived:

$$
\begin{aligned}
\mathcal{R}(a) & =\int_{0}^{\infty} \frac{\operatorname{sech}\left(\frac{\pi x}{2 a}\right)}{1+x^{2}} d x=2 a \sum_{k=1}^{\infty} \frac{(-1)^{k+1}}{1+(2 k-1) a} \\
& =\frac{1}{2}\left(\psi\left(\frac{3}{4}+\frac{1}{4 a}\right)-\psi\left(\frac{1}{4}+\frac{1}{4 a}\right)\right)
\end{aligned}
$$




$$
\begin{aligned}
& =\frac{2 a}{1+a} F\left(\frac{1}{2 a}+\frac{1}{2}, 1 ; \frac{1}{2 a}+\frac{3}{2} ;-1\right) \\
& =2 \int_{0}^{1} t^{1 / a}\left(1+t^{2}\right)^{-1} d t=\int_{0}^{\infty} e^{-x / a} \operatorname{sech}(x) d x .
\end{aligned}
$$

The first series representation or the $t$-integral can be used to establish a recurrence

$$
\mathcal{R}(a)=\frac{2 a}{1+a}-\mathcal{R}\left(\frac{a}{1+2 a}\right)
$$

while known relations for the digamma [Abramovitz and Stegun 70, Stromberg 81] can be used-with some symbolic care - to derive

$$
\begin{aligned}
& \mathcal{R}(a)=\frac{\pi}{2} \sec \frac{\pi}{2 a} \\
& -2 \frac{a^{2}\left(1+8 a-106 a^{2}+280 a^{3}+9 a^{4}\right)}{1-12 a+25 a^{2}+120 a^{3}-341 a^{4}-108 a^{5}+315 a^{6}} \\
& +C(a)
\end{aligned}
$$

where

$$
C(a)=\frac{1}{2} \sum_{n \geq 1}(\zeta(2 n+1)-1) \frac{(3 a-1)^{2 n}-(a-1)^{2 n}}{(4 a)^{2 n}}
$$

is a "rational-zeta" series as analyzed in [Borwein et al. $00]$. Note that this representation of $\mathcal{R}(a)$, while allowing rapid convergence for some $a$, has sec poles, some of which are cancelled by the rational function. In any case we require $a>1 / 9$ for convergence of the rational-zeta sum; however, as a computational matter, the recurrence relation above can generally be used to force convergence of such a rational-zeta series.

The hypergeometric form $(4-1)$ is of special interest, for there is the Gauss continued fraction for $F(\gamma, 1 ; 1+$ $\gamma ;-1)$, as discussed in [Borwein and Bailey 03]. Without belaboring the reader with details, we simply give a relevant $\mathrm{RCF}$ form, which will later prove useful in convergence analysis:

$$
\begin{gathered}
F(\gamma, 1 ; 1+\gamma ;-1)=\left[\alpha_{1}, \alpha_{2}, \ldots\right] \\
=\frac{1}{\alpha_{1}+\frac{1}{\alpha_{2}+\frac{1}{\alpha_{3}+\frac{1}{\alpha_{4}+} \cdot}}}
\end{gathered}
$$

where $\alpha_{1}=1$, and

$$
\begin{gathered}
\left.\alpha_{n}=((n-1) / 2) !\right)^{-2} \gamma(n-1+\gamma) \prod_{j 1}^{(n-3) / 2}(j+\gamma)^{2}, \\
n=3,5,7, \ldots \\
\alpha_{n}=\frac{1}{\gamma}(n / 2-1) !^{2}(n-1+\gamma) \prod_{j 1}^{n / 2-1}(j+\gamma)^{-2}, \\
n=2,4,6, \ldots .
\end{gathered}
$$

An interesting aspect of the formal analysis is based upon the first sech-integral form for $\mathcal{R}(a)$. Expanding said integral formally, and using a representation of the Euler number $E_{2 n}=(-1)^{n} \int_{0}^{\infty} \operatorname{sech}(\pi x / 2) x^{2 n} d x$, one obtains

$$
\mathcal{R}(a) \sim \sum_{n \geq 0} E_{2 n} a^{2 n+1},
$$

where we are indicating an asymptotic series of zero radius of convergence. (It is a classic theorem of Borel [Stromberg 81, Borwein et al. 04] that, for every real sequence $\left(a_{n}\right)$, there exists a $C_{\infty}$ function $f$ on $R$ such that $f^{(n)}(0)=a_{n}$.) It is possible to give expressions for the asymptotic error, such as

$$
\left|\mathcal{R}(a)-\sum_{n 1}^{N-1} E_{2 n} a^{2 n+1}\right| \leq\left|E_{2 N}\right| a^{2 N+1}
$$

from [Borwein et al. 04, Borwein et al. 03], but it is also interesting to employ Padé approximants to the formal asymptotic series. It turns out that the oft-stated success of the Padé approach is exemplified well in our case. Indeed, if one takes the unique $(3,3)$ Padé form (meaning numerator and denominator of $\mathcal{R}(a) / a$ each have degree 3 in the variable $a^{2}$ ), we obtain

$$
\mathcal{R}(a) \approx a \frac{1+90 a^{2}+1433 a^{4}+2304 a^{6}}{1+91 a^{2}+1519 a^{4}+3429 a^{6}} .
$$

Even this simple approximant is remarkably good for small $a$ (e.g., yielding $\mathcal{R}(1 / 10) \approx 0.09904494$, which is correct to the implied precision). For something like $\mathcal{R}(1 / 2)$ and the $(30,30)$ Padé approximant - so that numerator and denominator have degree 30 in $a^{2}$-one obtains 4 good digits. Though the convergence rate is slower for larger $a$, the method does give rapid means of, say, graphing the $R$ function to reasonable precision.

Having briefly discussed a formal expansion at $a=0$, can one establish an asymptotic form for large $a$ ? The answer is yes - except that, through a typical development for asymptotic forms, we are rewarded with more, namely a convergent expansion for all $a>1$. Using our second sech integral $\mathcal{R}(a)=\int_{0}^{\infty} e^{-x / a} \operatorname{sech} x d x$, we can 
again use the Euler numbers and known Hurwitz-zeta evaluations of sech-power integrals for odd powers to obtain a convergent series valid at least for real $a>1$ :

$$
\begin{aligned}
\mathcal{R}(a) & =\frac{\pi}{2} \sec \left(\frac{\pi}{2 a}\right)-2 \sum_{m \in D^{+}} \frac{\eta(m+1)}{a^{m}} \\
& =2 \sum_{k \geq 0} \eta(k+1)\left(\frac{-1}{a}\right)^{k}
\end{aligned}
$$

where $D^{+}$denotes the positive odd integers and $\eta(s):=$ $1 / 1^{s}-1 / 3^{s}+1 / 5^{s}-\ldots$ (this standard alternating zeta function $\eta$ is not to be confused with Ramanujan's $\eta$ parameter). Remarkably, we find that the leading terms for large $a$ involve the Catalan constant $G:=\eta(2)$ as

$$
\mathcal{R}(a)=\frac{\pi}{2}-\frac{2 G}{a}+\frac{\pi^{3}}{16 a^{2}}-\ldots,
$$

a development certainly difficult to infer by casual inspection of the Ramanujan fraction. (Even the asymptote $\mathcal{R}(\infty)=\pi / 2$ is difficult to so infer, although such is clear from various of the previous representations for $\mathcal{R}(a)$.)

Using recurrence relations together with various expansions, we have derived certain results pertaining to the derivatives of $\mathcal{R}$, notably

$$
\begin{aligned}
& \mathcal{R}^{\prime}(1)=8(1-G), \\
& \mathcal{R}^{\prime}(1 / 2)=\pi^{2} / 24 .
\end{aligned}
$$

To close this section we note that a peculiar property of the digamma $\psi$ leads to an exact evaluation of the imaginary part of $\mathcal{R}(a)$ when $a$ lies on the circle $C_{1 / 2}:=\{z:|z-1 / 2|=1 / 2\}$ in the complex plane. Because imaginary parts of certain digamma evaluations can be expressed in closed form [Abramovitz and Stegun 70, Stromberg 81], we have, for $a \in C_{1 / 2}$ and $y:=i(1-1 / a)$ (which $y$ is therefore real),

$$
\operatorname{Im}(\mathcal{R}(a))=-\frac{1}{y}+\frac{\pi}{2} \operatorname{cosech}\left(\frac{\pi y}{2}\right) .
$$

Thus, we have an elementary form for $\operatorname{Im}(\mathcal{R})$ on a certain continuum set. Admittedly we have not yet discussed convergence for complex parameters in depth; we do that later in Section 9.

\section{THE $\mathcal{R}$ FUNCTION AT RATIONAL ARGUMENTS}

From the first summation in the $\mathcal{R}(a)$-relations in the beginning of Section 4 , we have, for positive integers $p, q$,

$$
\mathcal{R}\left(\frac{p}{q}\right)=2 p\left(\frac{1}{q+p}-\frac{1}{q+3 p}+\frac{1}{q+5 p}-\ldots\right),
$$

which is essentially in the form of a particular $L$-function. One way to evaluate $L$-functions in finite form is to apply Fourier-transform techniques to pick out the correct terms from a general logarithmic series. (We note that an equivalent, elementary form for the digamma at rational arguments is a celebrated result of Gauss.) In our case

$$
\begin{aligned}
& \mathcal{R}\left(\frac{p}{q}\right)=\sum_{0<\operatorname{odd} k<4 p} e^{-2 \pi i k(q+p) /(4 p)} \\
& \quad \times\left(-\log \left(1-e^{2 \pi i k /(4 p)}\right)-\sum_{n=1}^{q+p-1} e^{2 \pi i k n /(4 p)} / n\right) .
\end{aligned}
$$

After various simplifications, especially forcing everything to be real-valued, we arrive at a finite series in fundamental numbers, namely

$$
\begin{gathered}
\mathcal{R}\left(\frac{p}{q}\right)=-2 p \sum_{n=1}^{p+q-1} \frac{1}{n}\left(\delta_{n \equiv p+q \bmod 4 p}-\delta_{n \equiv 3 p+q \bmod 4 p}\right) \\
-2 \sum_{0<\text { odd } k<2 p}\left(\cos \left(\frac{(p+q) k \pi}{2 p}\right)\right. \\
\left.\times \log \left(2 \sin \left(\frac{\pi k}{4 p}\right)\right)-\pi\left(\frac{1}{2}-\frac{k}{4 p}\right) \sin \left(\frac{(p+q) k \pi}{2 p}\right)\right) .
\end{gathered}
$$

Note that when $q=1$ - that is, when we seek $\mathcal{R}(p)$ for some integer $p$-the first, rational sum vanishes. The manifestly finite series (5-1) (of $O(p+q)$ total terms) leads quickly to exact evaluations such as

$$
\mathcal{R}(1 / 4)=\frac{\pi}{2}-\frac{4}{3}, \quad \mathcal{R}(1 / 3)=1-\log 2,
$$

$\mathcal{R}(1 / 2)=2-\pi / 2, \quad \mathcal{R}(2 / 3)=4-\frac{\pi}{\sqrt{2}}-\sqrt{2} \log (1+\sqrt{2})$,

$$
\mathcal{R}(1)=\log 2, \quad \mathcal{R}(3 / 2)=\pi+\sqrt{3} \log (2-\sqrt{3}),
$$

$\mathcal{R}(2)=\sqrt{2}\left\{\frac{\pi}{2}-\log (1+\sqrt{2})\right\}, \quad \mathcal{R}(3)=\frac{\pi}{\sqrt{3}}-\log 2$,

and, of course, many other attractive forms. It is not hard to establish from the finite series $(5-1)$ that, for positive integer $q$, one has

$$
\begin{gathered}
\mathcal{R}(1 / q)=\text { rational }+(-1)^{(q-1) / 2} \log 2, q \text { odd }, \\
\mathcal{R}(1 / q)=\text { rational }+(-1)^{q / 2} \pi / 2, q \text { even. }
\end{gathered}
$$

These facts can also be derived on knowledge of $\mathcal{R}(1)=$ $\log 2, \mathcal{R}(1 / 2)=2-\pi / 2$, and the recurrence

$$
\mathcal{R}\left(\frac{1}{q}\right)=\frac{2}{q-1}-\mathcal{R}\left(\frac{1}{q-2}\right) .
$$


On the other hand, one of the more alluring integerargument evaluations involves the golden mean $\tau=$ $(1+\sqrt{5}) / 2$, since

$$
\mathcal{R}(5)=\frac{\pi}{\sqrt{\tau \sqrt{5}}}+\log 2-\sqrt{5} \log \tau,
$$

although such evaluations - stemming from (5-1) - can involve quite delicate symbolic manipulations. We have not analyzed the possibility of evaluating $\mathcal{R}(a)$ for irrational $a$ via the expedient of approximating $a$ first via high-resolution rationals and then using (5-1), although such a development would be of both computational and theoretical interest.

Incidentally, armed with exact knowledge of $\mathcal{R}(p / q)$ we find some interesting Gauss-fraction results, in the form of rational multiples of $F(\gamma, 1 ; 1+\gamma ;-1)=$ $\left[\alpha_{1}, \alpha_{2}, \ldots\right]$; for example, on the basis of (4-2) we have

$$
\mathcal{R}(1)=\log 2=\frac{1}{1+\frac{1}{2+\frac{1}{3+\frac{1}{1+\ddots}}}} .
$$

But alas, the beginnings of this continued fraction are misleading; subsequent elements $a_{n}$ go according to

$$
\log 2=\left[1,2,3,1,5, \frac{2}{3}, 7, \frac{1}{2}, 9, \frac{2}{5}, \ldots\right],
$$

since $\alpha_{n}=n$ and $\alpha_{n}=4 / n$ as $n$ is odd and even, respectively. Similarly, one can derive

$$
2-\log 4=\left[1^{3}, r_{2}, 2^{3}, r_{4}, 3^{3}, r_{6}, 4^{3}, \ldots\right],
$$

where the even-indexed fraction elements $r_{2 n}$ are certain rationals. Though these RCFs are not SCFs (integer elements), the growths of the $\alpha_{n}$ still provide a clue to the convergence rate, which we study in a subsequent section.

\section{TRANSFORMATION OF $\mathcal{R}_{1}(a, b)$}

There is one remaining avenue that must be traversed in order to provide a uniformly rapid evaluation scheme for $\mathcal{R}_{1}(a, b)$ with positive real $a$ and $b$. We have mentioned that the sech-elliptic series (3-1) (also (3-2)) will converge slowly when $b \approx a$, yet in Sections 4 and 5 we successfully addressed the case $b=a$. So, we now proceed to establish a series representation for the case $b<a$ with $b$ very near to $a$. We employ the wonderful fact that sech is its own Fourier transform, in that

$$
\int_{-\infty}^{\infty} e^{i \gamma x} \operatorname{sech}(\lambda x) d x=\frac{\pi}{\lambda} \operatorname{sech} \frac{\pi \gamma}{2 \lambda} .
$$

Using this relation, one can perform a Poisson transform of the sech-elliptic series (3-1). The success of the transform depends on knowing the 2-parameter integral

$$
I(\lambda, \gamma)=\int_{-\infty}^{\infty} \frac{\operatorname{sech} \lambda x}{1+x^{2}} e^{i \gamma x} d x
$$

One may write down a differential equation with source

$$
-\frac{\partial^{2} I}{\partial \gamma^{2}}+I=\frac{\pi}{\lambda} \operatorname{sech} \frac{\pi \gamma}{2 \lambda}
$$

and solve this - after some delicate machinations - to yield

$$
I(\lambda, \gamma)=\frac{\pi}{\cos \lambda} e^{-\gamma}+\frac{2 \pi}{\lambda} \sum_{d \in D^{+}} \frac{(-1)^{(d-1) / 2} e^{-\pi d \gamma /(2 \lambda)}}{1-\pi^{2} d^{2} /\left(4 \lambda^{2}\right)},
$$

where $D^{+}$denotes the positive odd integers. In the event that $\lambda=\pi D / 2$ for some odd $D$, the $1 /$ cos pole conveniently cancels a corresponding pole in the summation, and the result can be inferred either by avoiding $d=D$ in the sum and inserting a precise residual term

$$
\Delta I=\pi(-1)^{(D-1) / 2} e^{-\gamma}(\gamma+1 / 2) / \lambda,
$$

or, more simply, by taking a numerical limit as $\lambda \rightarrow$ $\pi D / 2$. When $\gamma \rightarrow 0$, we can recover from the sum, via analytic relations for $\psi(z)$, the $\psi$-function form of the integral of $(\operatorname{sech} \lambda x) /\left(1+x^{2}\right)$. Via the Poisson transformation of (3-1), we thus obtain, for $0<b<a$,

$$
\begin{aligned}
\mathcal{R}_{1}(a, b)= & \mathcal{R}\left(\frac{\pi a}{2 \mathrm{~K}^{\prime}}\right)+\frac{\pi}{\cos \frac{\mathrm{K}^{\prime}}{a} \frac{1}{e^{2 \mathrm{~K} / a}-1}} \\
& +8 \pi a \mathrm{~K}^{\prime} \sum_{d \in D^{+}} \frac{(-1)^{(d-1) / 2}}{4 \mathrm{~K}^{\prime 2}-\pi^{2} d^{2} a^{2}} \frac{1}{e^{\pi d \mathrm{~K} / \mathrm{K}^{\prime}}-1},
\end{aligned}
$$

where again $k:=b / a, \mathrm{~K}:=\mathrm{K}(k), \mathrm{K}^{\prime}:=\mathrm{K}\left(k^{\prime}\right)$, and $D^{+}$denotes the positive odd integers. A similar Poisson transform can be obtained from (3-2) in the case $b>a$. Such transformations appear recondite, but we have achieved what we desired: convergence is rapid for $b \approx a$.

\section{CONVERGENCE RESULTS FOR REAL PARAMETERS}

For an RCF of the form $x=\left[a_{0}, a_{1}, \ldots\right]$ (so that each $a_{i}$ is nonnegative and real but not necessarily an integer), one has the usual recurrence relations for convergents

$$
\begin{aligned}
p_{n} & =a_{n} p_{n-1}+p_{n-2}, \\
q_{n} & =a_{n} q_{n-1}+q_{n-2},
\end{aligned}
$$


with $\left(p_{0}, p_{-1}, q_{0}, q_{-1}\right):=\left(a_{0}, 1,1,0\right)$. We also have the approximation rule

$$
\left|x-\frac{p_{n}}{q_{n}}\right|<\frac{1}{q_{n} q_{n+1}}
$$

so that convergence rates can be bounded by virtue of the growth of $q_{n}$. One may iterate the recurrence in various ways, obtaining, for example,

$$
q_{n}=\left(1+a_{n} a_{n-1}+a_{n} / a_{n-2}\right) q_{n-2}-\left(a_{n} / a_{n-2}\right) q_{n-4}
$$

a relation involving all even or all odd indices on $q$. An immediate application is the following theorem:

Theorem 7.1. For the RCF form of the Gauss continued fraction, $F(\gamma, 1 ; 1+\gamma ;-1)=\left[\alpha_{1}, \alpha_{2}, \ldots\right]$, and for $\gamma>$ $1 / 2$ we have

$$
\left|F-\frac{p_{n}}{q_{n}}\right|<\frac{c}{8^{n / 2}},
$$

where $c$ is an absolute constant.

Remark 7.2. One can likely obtain sharper bounds, or better $\gamma$-dependent bounds. We intend here just to show geometric convergence; i.e., we intend to show that the number of good digits grows at least linearly in the number of iterates. Also note that, for the $\mathcal{R}(a)$ evaluation of interest, $\gamma=1 / 2+1 /(2 a)$; thus, the condition on $\gamma$ is natural.

Proof: From the element assignments following (4-2) we have

$$
\begin{gathered}
\alpha_{n} \alpha_{n-1}=\frac{4}{(n-1)^{2}}(n-1+\gamma)(n-2+\gamma), n \text { odd }>1, \\
\alpha_{n} \alpha_{n-1}=\frac{1}{(n / 2-1+\gamma)^{2}}(n-1+\gamma)(n-2+\gamma), n \text { even. }
\end{gathered}
$$

We also have $q_{1}=1$ and $q_{2}=1+1 / \gamma>2$ so that, for sufficiently large $n$, we have

$$
\alpha_{n} \alpha_{n-1}+1>4 \quad \text { or } \quad 2
$$

as $n$ is odd or even, respectively. From $q_{n}>\left(\alpha_{n} \alpha_{n-1}+\right.$ 1) $q_{n-2}$ the desired bound follows.

It is appropriate here to mention a clever computational acceleration for Gauss continued fractions, as described in [Borwein and Bailey 03, Andrews et al. 99, Lorentzen and Waadeland 92]. Consider the previously displayed Gauss continued fraction $\log 2=$
$[1,2,3,1,5,2 / 3, \ldots]$. Generally a "tail" $t_{N}$ of this construct, meaning a subfraction starting from the $N$-th element, runs like so:

$$
t_{N}:=\frac{1}{\frac{4}{N}+\frac{1}{N+1+\frac{1}{\frac{4}{N+2}+\frac{1}{N+3+} \cdot}}} .
$$

But - and here is the clever idea from the literature - this tail $t_{N}$ should be near to the periodic continued fraction $[4 / N, N, 4 / N, N, \ldots]=N(\sqrt{2}-1) / 2$. This suggests that, if we are evaluating the Gauss continued fraction and we stop at element $4 / N$, this one element should be replaced with $2(1+\sqrt{2}) / N$. Indeed, in our own numerical experiments this expedient always adds a few digits of precision. What is more, as suggested in [Lorentzen and Waadeland 92], there are higher-order manifestations of this idea, e.g., the use of longer periods for the tail subfraction. As the reference shows via experimentation, the acceleration can become significant. Note also that our companion treatment [Borwein and Crandall 03] describes a similar speedup for the $\mathcal{R}$ continued fraction itself, even when parameters are allowed to become complex.

Now we move on to the convergence of the RCF arising from Ramanujan's original construct, namely

$$
\frac{a}{\mathcal{R}_{1}(a, b)}=\left[A_{0} ; A_{1}, A_{2}, A_{3}, \ldots\right],
$$

with the $A_{i}$ defined as in Section 2. It turns out that the $q_{n}$ convergents consist of linear combinations of terms $a^{i} b^{j}$ where $i$ and $j$ are even integers and that certain terms with explicit coefficients can be isolated, leading to

$$
q_{n} \geq 1+\frac{b^{n-2}}{a^{n}} \prod_{m \text { even }}^{n}(1-1 / m)^{2}>1+\frac{1}{2 n} \frac{b^{n-2}}{a^{n}}
$$

$n$ even,

$$
q_{n} \geq 1 / b^{2}+\frac{a^{n-1}}{b^{n+1}} \prod_{m \text { even }}^{n-1}(m /(m+1))^{2}>1 / b^{2}+\frac{1}{n} \frac{a^{n-1}}{b^{n+1}}
$$

$n$ odd.

Such observations lead to a convergence theorem for the original Ramanujan construct:

Theorem 7.3. For the Ramanujan $R C F, a / \mathcal{R}_{1}(a, b)=$ $\left[A_{0} ; A_{1}, A_{2}, A_{3}, \ldots\right]$, we have for positive reals $b>a$

$$
\left|\frac{a}{\mathcal{R}_{1}(a, b)}-\frac{p_{n}}{q_{n}}\right|<\frac{2 n b^{4}}{(b / a)^{n}},
$$


while for positive reals $a>b$ we have

$$
\left|\frac{a}{\mathcal{R}_{1}(a, b)}-\frac{p_{n}}{q_{n}}\right|<\frac{n b / a}{(a / b)^{n}} .
$$

Remark 7.4. Again, it should be possible to prove sharper bounds, our motive here being merely to establish essential geometric convergence of the literal continued fraction when $a$ and $b$ are not near each other.

Proof: The given approximation bounds follow directly upon inspection of the products $q_{n} q_{n+1}$.

As we previously have intimated, convergence for $a=b$ is slow. What we can prove is the following:

Theorem 7.5. For real $a>0$ the Ramanujan $R C F$, $a / \mathcal{R}(a)$, has

$$
\left|\frac{a}{\mathcal{R}(a)}-\frac{p_{n}}{q_{n}}\right|<\frac{c(a)}{n^{h(a)}},
$$

where $c(a)$ and $h(a)$ are $n$-independent, positive constants. The exponent $h(a)$ can be taken to be $c_{0} \min \left(1,4 \pi^{2} / a^{2}\right)$ where the constant $c_{0}$ is absolute.

Remark 7.6. Note that the convergence bound is computationally poor; still, as we have noted, convergence does occur. The relevant exponent $h(a)$ could be sharpenedor made more explicit — with more work; we only exhibit the theorem for theoretical completeness. Indeed, for $a=b$, or even $a \approx b$, we have many other rapidly convergent options.

Proof: With a view to induction, assume that for some constants ( $n$-independent) $d(a)$ and $g(a)$ and for $n \in$ $[1, N-1]$, we have $q_{n}<d n^{g}$. Note that the element asymptotics following $(2-1)$ mean that $A_{n}>f(a) / n$ for an $n$-independent $f$. Then we have a bound for the next $q_{N}:$

$$
q_{N}>\frac{f}{N} d(N-1)^{g}+d(N-2)^{g} .
$$

Using the fact that, for $g<1,0<x \leq 1 / 2$, we have $(1-x)^{g}>1-g x-g x^{2}$; the constants $d$ and $g$ can evidently be arranged such that $q_{N}>d N^{g}$ and the induction goes through.

To clarify the import of the above theorems, consider the following: the Gauss continued fraction for $\mathcal{R}(a)$ exhibits (at least) geometric convergence, as does the original Ramanujan form $\mathcal{R}(a, b)$ when $a / b$ or $b / a$ is significantly greater than unity. When $a=b$, we do have convergence although, as suggested by Theorem 7.5, the convergence is far below geometric.

\section{A UNIFORMLY CONVERGENT ALGORITHM}

We are now in a position to establish a complete algorithm for evaluating the original Ramanujan AGM continued fraction $\mathcal{R}_{\eta}(a, b)$ for positive real parameters. The convergence is uniform, in that for any positive real triple $\eta, a, b$ we expect rapid convergence in the sense of $D$ good digits in less than $c D$ computational iterations, where $c$ is an absolute constant independent of the magnitudes of $\eta$, $a$, and $b$. (Here, by iterations we mean either continuedfraction recurrence steps or series-summand additions.)

\section{Algorithm 8.1. (Algorithm for evaluation of $\mathcal{R}_{\eta}(a, b)$ with real $\eta, a, b>0$.)}

0 . Observe that $\mathcal{R}_{\eta}(a, b)=\mathcal{R}_{1}(a / \eta, b / \eta)$, so that with impunity we may assume $\eta=1$ and subsequently evaluate only $\mathcal{R}_{1}$

1. If $(a / b>2$ or $b / a>2)$, return the original continued fraction (1-1), or equivalently (2-1);

2. If $(a=b)\{$

if ( $a=p / q$ rational), return finite form (5-1); else return the Gauss RCF (4-2) or rational-zeta form $(4-1)$ or $(4-3)$

or some other scheme such as rapid $\psi$ computations, etc.;

\}

3. if $(b<a)\{$

if ( $b$ is not too close to $a$ ), return sech-elliptic result $(3-1)$;

else return Poisson-transform result (6-1); \}

4. (Here, we must have $b>a$ ) Return, as in (1-2), $2 \mathcal{R}_{1}((a+b) / 2, \sqrt{a b})-\mathcal{R}_{1}(b, a)$.

It is an implicit tribute to the ingenuity of Ramanujan that the final algorithm step allows the entire procedure to go through for any positive real parameters. One could avoid Step 4 by invoking a Poisson transformation of (3-2), but the Ramanujan AGM identity simplifies the procedure.

\section{COMPLEX PARAMETERS}

The issue of complex parameters $a, b$, and $\eta$ is profound, as we have discovered via both theoretical forays and extensive numerical experimentation. A companion treatment [Borwein and Crandall 03] deals with convergence issues, with theorems and conjectures we summarize below, where we remind ourselves that convergence of a 
complex continued fraction is often interpreted in a modern way as convergence of the $p_{n} / q_{n}$ on the extended complexes $\hat{\mathcal{C}}:=\mathcal{C} \cup\{\infty\}$ (so that divergence in such cases must be oscillatory, e.g., bifurcated or chaotic):

- The continued fraction $\mathcal{R}(a):=\mathcal{R}_{1}(a, a)$ converges on $\mathcal{C}$ (i.e., to a finite complex value) for all $a$ not purely imaginary, i.e., $a^{2} \notin(-\infty, 0)$.

- The continued fraction $\mathcal{R}_{1}(a, b)$ converges on $\hat{\mathcal{C}}$ for all $|a| \neq|b|$. It is proven (see [Borwein and Crandall 03]) — via a dynamical-recurrence equivalent of divergence - that the precise domain of convergence is

$$
\begin{aligned}
\mathcal{D}_{1}:=\{(a, b) \in \mathcal{C} \times \mathcal{C}:|a| & \neq|b| \text { or } \\
& \left.\left(a^{2}=b^{2} \notin(-\infty, 0)\right)\right\} .
\end{aligned}
$$

- There are direct means - e.g., inspection of even/odd fraction parts - to prove that the continued fraction $\mathcal{R}_{1}(a, b)$ does indeed diverge for certain instances of $|a|=|b|$, such as

$$
(a, b)=(i, i),(1, i),(\sqrt{i}, \sqrt{-i}),
$$

thus contradicting the claim of [Berndt 99b, p. 165] that the condition $\operatorname{Re}(a), \operatorname{Re}(b)>0$ suffices for convergence (and, therefore, the domain of validity of the AGM relation (2-1) comes into question).

- If $a / b$ is in the set $\mathcal{H}$ defined

$$
\mathcal{H}:=\{z \in \mathcal{C}:|\sqrt{z} /(1+z)|<1 / 2\}
$$

then, provably, all three of $\mathcal{R}_{1}(a, b), \mathcal{R}_{1}(b, a)$, and $\mathcal{R}_{1}((a+b) / 2, \sqrt{a b})$ converge on $\hat{\mathcal{C}}$.

- It is conjectured that, if $a / b \in \mathcal{H}$, then (with all three fractions already known to converge) the AGM relation $(2-1)$ holds.

Incidentally, the discovered restrictions on the convergence domain are "algorithmically unfortunate," if you will, because one might look longingly at the formal relation possibility (note the "? = signaling suspicion),

$$
\begin{aligned}
& \mathcal{R}_{1}\left(\sqrt{a b}+i \frac{a-b}{2}, \sqrt{a b}-i \frac{a-b}{2}\right) \\
&+\mathcal{R}_{1}\left(\sqrt{a b}-i \frac{a-b}{2},\right.\left.\sqrt{a b}+i \frac{a-b}{2}\right) \\
& \stackrel{?}{=} 2 \mathcal{R}_{1}\left(\sqrt{a b}, \frac{a+b}{2}\right),
\end{aligned}
$$

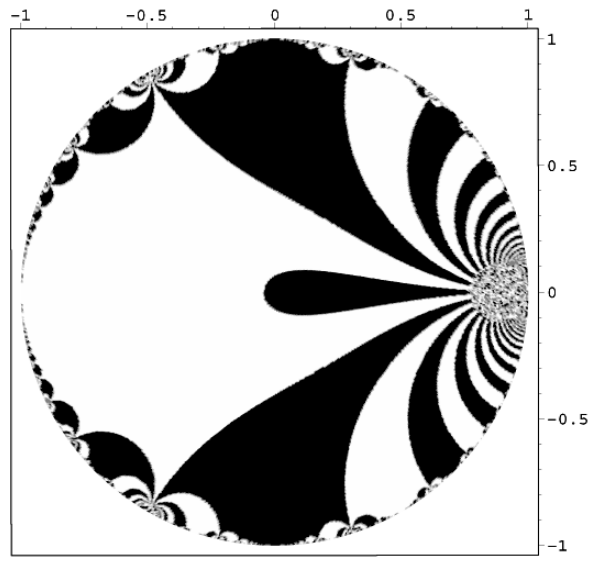

FIGURE 1. Where $\left|\theta_{2} / \theta_{3}\right|<1$ in the complex $q$-plane. Note that the real interval $(-c, 1)$ for some positive real $c$ is monochrome black.

by which one perhaps hopes to forge a "left-handed" AGM relation, possibly giving rise to new iterative algorithms. Alas, this reversed AGM relation is generally false. For one thing, in the case $a:=1+i$ and $b:=1-i$ the questionable relation is problematic because neither of $\mathcal{R}_{1}(1 \pm i, 1 \mp i)$ converges, yet the right-hand side $2 \mathcal{R}_{1}(\sqrt{2}, 1)$ does converge. Secondly, there are examples, such as $\mathcal{R}_{1}(2 i, 1)+\mathcal{R}_{1}(1,2 i) \neq 2 \mathcal{R}_{1}(1 / 2+i, 1+i)$, with all three fractions converging.

An observation that led us to realize such complications in regard to convergence is that, in the realparameter scenario for the sech identities of Theorem 2.1, one implicitly uses, for positive real $a \neq b$ and perforce for Jacobi parameter $q:=\min (a, b) / \max (a, b)$ in $[0,1)$, the fact that $\theta_{2} / \theta_{3}<1$. However, if one plots the complex $q$ such that this $\theta$-ratio has absolute value $<1$, one sees a frightfully complicated fractal structure in the complex $q$-plane, as shown in Figure 1. We also exhibit a related Figure 2, and all this in turn leads into the theory of modular forms [Borwein and Borwein 87]. It may well be true, however, that the sech identities (3-1) and (3-2) always hold for $|a|>|b|$ and $|a|<|b|$, respectively, with all $\mathcal{R}_{1}$ fractions converging. This supposition is what led us to the separate convergence study [Borwein and Crandall 03].

It is a classic and elementary observation that, for positive real $a$ and $b$, the arithmetic mean strictly dominates the geometric mean. A picturesque interpretation of such inequality for complex parameters is effected as follows. Note that

$$
a / b \in \mathcal{H} \text { implies } \mid(a+b) / 2)|>| \sqrt{a b} \mid \text {. }
$$




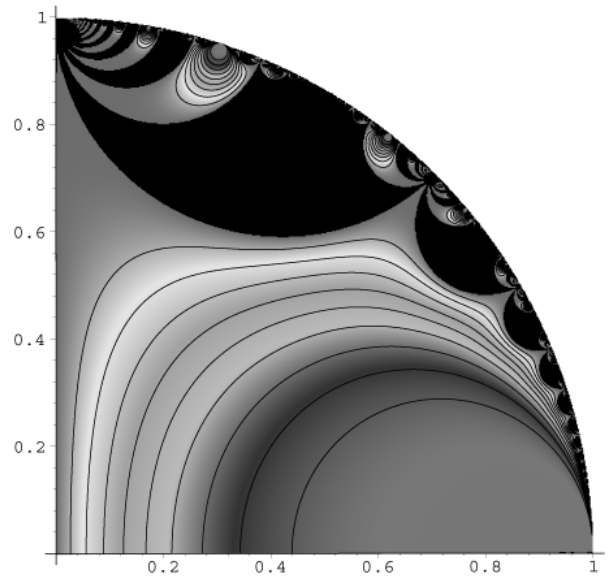

FIGURE 2. Values of $\left|\theta_{4} / \theta_{3}\right|$ (in first $q$-quadrant). The changes in gray-scale represent gradations of values between zero and one.

Now, $\mathcal{H}$ is actually the (open) exterior of a "cardioidknot" which in turn is the contour determined by the polar relation

$$
r^{2}+(2 \cos \phi-4) r+1=0
$$

in the complex plane. One can think of said contour as the fusion of two contours:

$$
r=2-\cos \theta \pm \sqrt{(1-\cos \theta)(3-\cos \theta)},
$$

that is, we fuse the orbits of the \pm instances, both for $\theta \in[0,2 \pi]$. One sees a small loop encompassing the origin, with left-intercept $\sqrt{8}-3+0 i$, and a wider contour

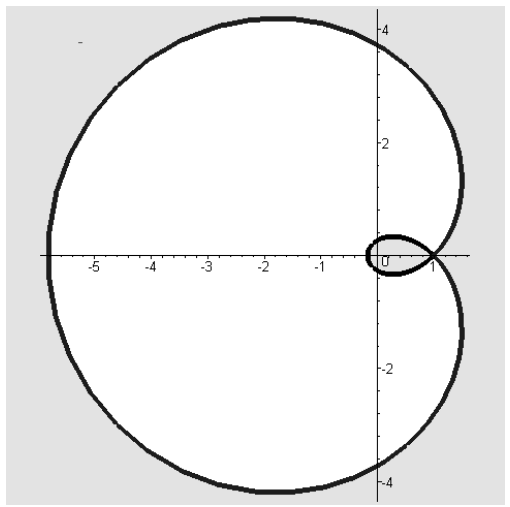

FIGURE 3. A cardioid-knot, the exterior $\mathcal{H}$ (in gray) of which knot is conjectured to ensure the truth of the Ramanujan AGM relation (1.2); we do know that the three relevant $\mathcal{R}_{1}$ fractions converge for $a / b \in \mathcal{H}$. whose left-intercept is $-3-\sqrt{8}+0 i$. So, $\mathcal{H}$ consists of all points outside the cardioid-knot, including the points in the inner lobe. One can call a point within said lobe an exterior point on the basis of the classical Jordan-curve rule: a point is outside a (smooth) contour if a ray to infinity from said point crosses the contour an even number of times. (See Figure 3.) We note also that for some pairs $(a, b)$ with $a / b$ on the knot itself - but not all such pairs (there is divergence for some such $(a, b)$ ) - the AGM relation still appears to hold.

\section{ACKNOWLEDGMENTS}

Thanks are due to David Bailey, Bruce Berndt, David Borwein, Joseph Buhler, Stephen Choi, William Jones, and Lisa Lorentzen for useful discussions. The research of the first author was supported by NSERC, the Canada Foundation for Innovation, and the Canada Research Chair Program.

\section{REFERENCES}

[Abramovitz and Stegun 70] Milton Abramowitz and Irene A. Stegun. Handbook of Mathematical Functions. New York: Dover, 1970.

[Andrews et al. 99] George E. Andrews, Richard Askey and Ranjan Roy. Special Functions. Cambridge, UK: Cambridge University Press, 1999.

[Berndt 99a] Bruce C. Berndt. Ramanujan's Notebooks, Part II. New York: Springer-Verlag, 1999.

[Berndt 99b] Bruce C. Berndt. Ramanujan's Notebooks, Part III. New York: Springer-Verlag, 1999.

[Borwein and Bailey 03] Jonathan M. Borwein and David H. Bailey. Mathematics by Experiment: Plausible Reasoning in the 21st Century. Wellesley, MA: A K Peters, Ltd., 2003 .

[Borwein et al. 04] Jonathan M. Borwein, David H. Bailey, and Roland Girgensohn. Experimentation in Mathematics: Computational Paths to Discovery. Wellesley, MA: A K Peters, Ltd., 2004.

[Borwein and Borwein 87] Jonathan M. Borwein and Peter B. Borwein. Pi and the AGM: A Study in Analytic Number Theory and Computational Complexity, CMS Series of Mongraphs and Advanced Books in Mathematics. New York: John Wiley \& Sons, 1987.

[Borwein et al. 00] Jonathan M. Borwein, David M. Bradley, and Richard E. Crandall. "Computational Strategies for the Riemann Zeta Function." Journal of Computational and Applied Mathematics 121 (2000), 247-296.

[Borwein et al. 03] Jonathan M. Borwein, Kwok-Kwong Stephen Choi, and Wilfrid Pigulla. "Continued Fractions as Accelerations of Series." Available from World Wide Web (http://www.cecm.sfu.ca/preprints/2003pp.html), 2003 . 
[Borwein and Crandall 03] J. Borwein and R. Crandall, "On the Ramanujan AGM Fraction, Part II: The ComplexParameter Case." Exp. Math. 13:3 (2004), 287-295.

[Henrici 91] P. Henrici. Applied and Computational Complex Analysis: Special Functions, Integral Transforms, Asymptotics, Continnued Fractions. New York: Wiley, 1991.

[Jones and Thron 80] W. Jones and W. Thron. Continued Fractions: Analytic Theory and Applications. Reading, MA: Addison-Wesley, 1980.
[Khintchine 64] A. Khintchine. Continued Fractions. Chicago: University of Chicago Press, 1964.

[Lorentzen and Waadeland 92] L. Lorentzen and H. Waadeland. Continued Fractions With Applications. Amsterdam: North-Holland, 1992.

[Stromberg 81] Karl R. Stromberg. An Introduction to Classical Real Analysis. Pacific Grove, CA: Wadsworth, 1981.

[Wall 48] H. S. Wall. Analytic Theory of Continued Fractions. New York: Van Nostrand, 1948.

J. Borwein, Faculty of Computer Science, Dalhousie University, Halifax, Nova Scotia B3H 1W5, Canada (jborwein@cs.dal.ca)

R. Crandall, Center for Advanced Computation, Reed College, Portland, OR 97202 (crandall@reed.edu)

G. Fee, Centre for Experimental and Constructive Mathematics (CECM) and Department of Mathematics, Simon Fraser University, Burnaby, BC V5A 1S6, Canada (gfee@cecm.sfu.ca)

Received June 17, 2003; accepted August 29, 2003. 